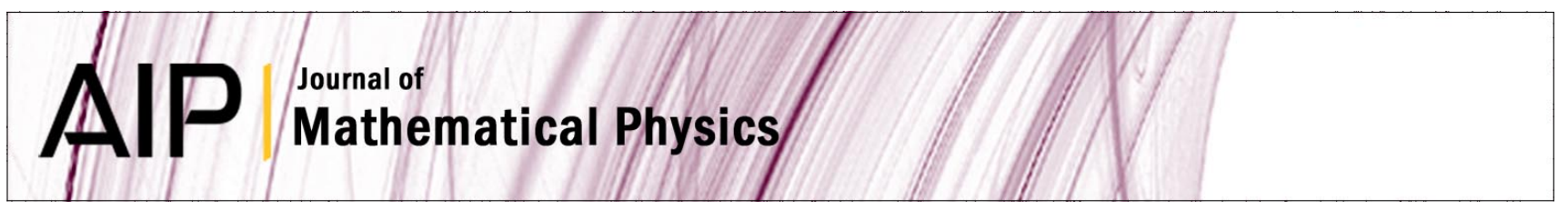

\title{
On the gravitational potential of modified Newtonian dynamics
}

Manuel Núñez

Citation: J. Math. Phys. 54, 082502 (2013); doi: 10.1063/1.4817858

View online: http://dx.doi.org/10.1063/1.4817858

View Table of Contents: http://jmp.aip.org/resource/1/JMAPAQ/v54/i8

Published by the AIP Publishing LLC.

\section{Additional information on J. Math. Phys.}

Journal Homepage: http://jmp.aip.org/

Journal Information: http://jmp.aip.org/about/about_the_journal

Top downloads: http://jmp.aip.org/features/most_downloaded

Information for Authors: http://jmp.aip.org/authors

\section{ADVERTISEMENT}

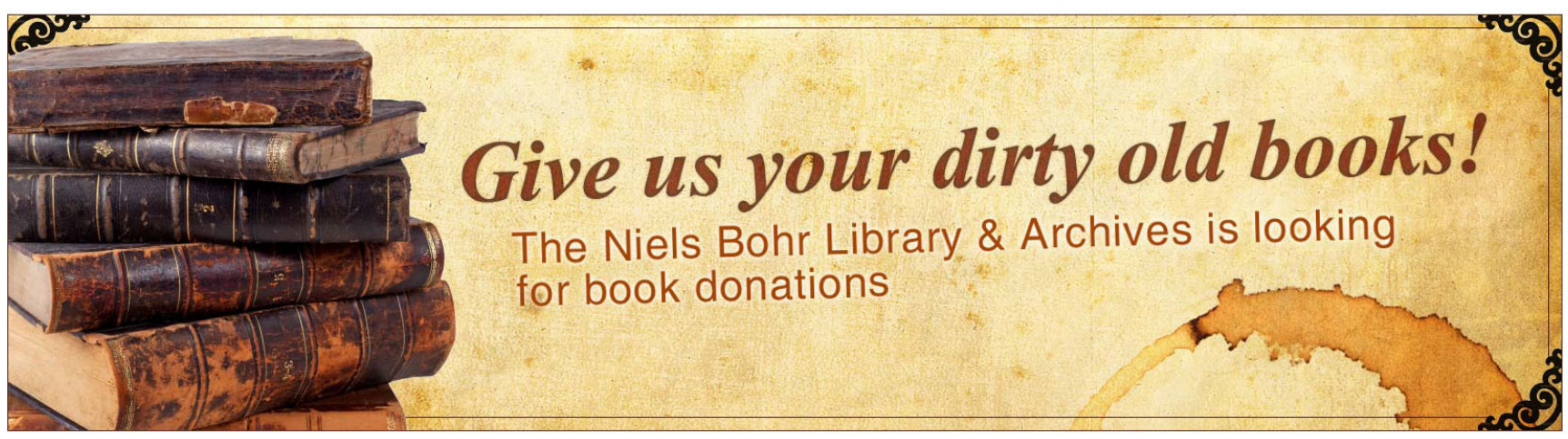




\title{
On the gravitational potential of modified Newtonian dynamics
}

\author{
Manuel Núñez ${ }^{\text {a) }}$ \\ Sección de Matemáticas and IMUVA, Universidad de Valladolid, 47011 Valladolid, Spain
}

(Received 11 February 2013; accepted 25 July 2013; published online 12 August 2013)

\begin{abstract}
The mathematical structure of the Poisson equation of Modified Newtonian Dynamics (MOND) is studied. The appropriate setting turns out to be an Orlicz-Sobolev space whose Orlicz function is related to Milgrom's $\mu$-function, where there exists existence and uniqueness of weak solutions. Since these do not have in principle much regularity, a further study is performed where the gravitational field is not too large, where MOND is most relevant. In that case the field turns out to be Hölder continuous. Quasilinear MOND is also analyzed. ( 2013 AIP Publishing LLC. [http://dx.doi.org/10.1063/1.4817858]
\end{abstract}

\section{INTRODUCTION}

Modified Newtonian Dynamics (MOND) was proposed by M. Milgrom ${ }^{1,2}$ as an explanation of the observed dynamical properties of galaxies without the need to invoke the presence of hidden (dark) matter. This hypothesis has been rather successful from a phenomenological viewpoint ${ }^{3}$ and given a fully relativistic foundation. ${ }^{4,5}$ Obviously objections have been raised, ${ }^{6-8}$ but on the whole this theory has emerged rather unscathed and is still an active subject of research. What seems to be absent is a mathematical study of the basic MOND-Poisson equation. Milgrom himself proved uniqueness of solutions by an essentially correct method, ${ }^{9}$ and several exact solutions were later found, ${ }^{10}$ but neither the general existence of solutions nor their regularity seems to have been studied so far. Recently, Milgrom proposed a simpler version of MOND. ${ }^{11}$

The essence of the MOND proposal in its simplest form is that the Newtonian gravitational field does not describe correctly the forces when the gradient is very small. This may be translated by substituting Poisson's equation for the gravitational potential $u$,

$$
\Delta u=4 \pi G \rho,
$$

by the equation

$$
\nabla \cdot\left(\mu\left(\frac{|\nabla u|}{a_{0}}\right) \nabla u\right)=4 \pi G \rho
$$

where $\mu(x)$ is an increasing function that behaves like $x$ when $x \rightarrow 0$, and tends to 1 when $x \rightarrow \infty$, so that the field is essentially Newtonian if $|\nabla u| \gg a_{0}$. The value of $a_{0}$ is $10^{-10} \mathrm{~m} \mathrm{~s}^{-2}$ and as usual $\rho$ is the material density. The effects of (2) will be more apparent at weak fields (e.g., in the far reaches of a galaxy, affecting its rotation curves; this was the initial motivation of MOND). There exist more accessible points of weak field, such as the neutral point between two massive bodies, a fact which has been used to propose a test method. ${ }^{12}$ Unfortunately, the mathematical difficulties of (2) are considerable; not only it is a nonlinear equation, but also it is not elliptic in the presence of critical points of the field. It has been solved analytically in a few cases, and numerically in several others, but no general theorem about it has been stated. Our arguments will hold for a wide variety

a) Telephone: 34983423924. Electronic mail: mnjmhd@am.uva.es 
of functions $\mu$, but we will use for concretion the form

$$
\mu(x)=\frac{x}{1+x},
$$

which has been used rather successfully to model galaxy rotation. After a rescaling, we may take $a_{0}$ $=1$ and absorb $4 \pi G$ into the density to obtain

$$
\nabla \cdot(\mu(|\nabla u|) \nabla u)=\rho .
$$

It will be useful to define associated functions which approximate $\mu$ in areas of weak field: those are

$$
\mu_{\epsilon}(x)=\epsilon x+(1-\epsilon) \mu(x) .
$$

\section{THE MAIN THEOREM}

We will work in a bounded open set $\Omega$ with smooth boundary, and use some spaces of Orliczintegrable functions defined in $\Omega$. For Orlicz spaces the most classical (and still the best) reference is Ref. 13; Orlicz-Sobolev spaces may be consulted, e.g., in Refs. 14 and 15. We will recall the basic facts. Let $M$ be a continuous, positive, increasing, convex function defined in $[0, \infty)$ such that

$$
\begin{aligned}
& \lim _{x \rightarrow \infty} \frac{M(x)}{x}=\infty, \\
& \lim _{x \rightarrow 0} \frac{M(x)}{x}=0 .
\end{aligned}
$$

Assume that $M$ satisfies the $\Delta_{2}$ property: there exist $k, x_{0}$ such that

$$
M(2 x) \leq k M(x) \quad \forall x \geq x_{0} .
$$

Then,

$$
L^{M}(\Omega)=\left\{f: \Omega \rightarrow \mathbb{C}: \int_{\Omega} M(|f(x)|) d x<\infty\right\}
$$

is a separable Banach space, contained in $L^{1}(\Omega)$. The complementary function

$$
N(x)=\sup _{y \geq 0}(y|x|-M(y))
$$

satisfies the same properties as $M$, except for $\Delta_{2}$; this holds if $M$ satisfies that there exist $r>1, x_{0}$ $\geq 0$ such that

$$
M(x) \leq \frac{1}{2 r} M(r x) \quad \forall x \geq x_{0} .
$$

If (11) holds, then $L^{M}(\Omega)$ is reflexive. In fact every continuous linear functional on $L^{M}(\Omega)$ has the form

$$
u \rightarrow \int_{\Omega} v u d x
$$

for a certain $v \in L^{N}(\Omega)$. The norms in these spaces are rather cumbersome to define: see Ref. 13 . The inequality

$$
\left|\int_{\Omega} u v d x\right| \leq\|u\|_{L^{M}}\|v\|_{L^{N}}
$$

holds.

We will consider the functions

$$
M_{\epsilon}(x)=\mu_{\epsilon}(x) x^{2}=\epsilon x^{3}+(1-\epsilon) \frac{x^{3}}{1+x} .
$$


It is easy to see that (8) holds for $k=8$ and (11) for $r=2$. Thus, the Banach space $L^{M}(\Omega)$ is separable and reflexive.

The Orlicz-Sobolev space $W^{1, M}(\Omega)$ is (roughly) formed by the functions $u$ such that $u, \nabla u \in$ $L^{M}(\Omega)$, with norm

$$
\|u\|_{W^{1, M}}=\left(\|u\|_{L^{M}}^{2}+\|\nabla u\|_{L^{M}}^{2}\right)^{1 / 2} .
$$

The closure of $\mathcal{C}_{c}^{\infty}(\Omega)$ in $W^{1, M}(\Omega)$ is denoted by $W_{0}^{1, M}(\Omega)$. There the norm may be reduced to the term in $\nabla u$ in (15).

Lemma 1. Assume that $u, v \in W^{1, M_{\epsilon}}(\Omega)$. The integral

$$
\left(\mu_{\epsilon}(|\nabla u|) \nabla u, \nabla v\right)=\int_{\Omega} \mu_{\epsilon}(|\nabla u|) \nabla u \cdot \nabla v d x
$$

is finite for any $\epsilon \in[0,1]$.

Proof. Obviously

$$
\int_{\Omega}\left|\mu_{\epsilon}(|\nabla u|) \nabla u \cdot \nabla v\right| d x \leq \int_{\Omega} \frac{M_{\epsilon}(|\nabla u|)}{|\nabla u|}|\nabla v| d x .
$$

Since

$$
N_{\epsilon}\left(\frac{M_{\epsilon}(x)}{x}\right) \leq M_{\epsilon}(x)
$$

(see Ref. 14, p. 137), the function $\mu_{\epsilon}(|\nabla u|) \nabla u$ lies in $L^{N_{\epsilon}}(\Omega)$. The result follows from (13).

We will denote by $A_{\epsilon}$ the operator

$$
\begin{array}{r}
A_{\epsilon}: W^{1, M}(\Omega) \rightarrow L^{N}(\Omega) \\
A_{\epsilon}(u)=\mu_{\epsilon}(|\nabla u|) \nabla u .
\end{array}
$$

Lemma 2. The operator $A_{\epsilon}$ is hemicontinuous: for $u, v, w \in W^{1, M}(\Omega)$, the mapping $\mathbb{R} \rightarrow \mathbb{C}$

$$
\lambda \rightarrow\left(A_{\epsilon}(u+\lambda v), \nabla w\right),
$$

is continuous.

Proof. Since obviously the mapping

$$
\lambda \rightarrow \mu_{\epsilon}(|\nabla(u+\lambda v)(\mathbf{x})|) \nabla(u+\lambda v)(\mathbf{x}) \cdot \nabla w(\mathbf{x})
$$

is continuous for every point $\mathbf{x} \in \Omega$, we only need to bound the integrand by an integrable function uniformly in a neighbourhood of every $\lambda$ to apply Lebesgue's theorem on the continuity of parametric integrals. Since the function

$$
F_{\epsilon}: x \rightarrow x \mu_{\epsilon}((x)
$$

is convex and increasing, we conclude

$$
F_{\epsilon}(|\nabla u+\lambda \nabla v|) \leq F_{\epsilon}(|\nabla u|+|\lambda||\nabla v|) \leq \frac{1}{2} F_{\epsilon}(2|\nabla u|)+\frac{1}{2} F_{\epsilon}(2|\lambda||\nabla v|) .
$$

If we take $\lambda$ with $|\lambda| \leq k$, we obtain

$$
F_{\epsilon}(|\nabla u+\lambda \nabla v|) \leq \frac{1}{2} F_{\epsilon}(|\nabla 2 u|)+\frac{1}{2} F_{\epsilon}(|\nabla 2 k v|) .
$$

Since both $2 u$ and $2 k v$ lie in the space $W^{1, M_{\epsilon}}(\Omega)$, the conclusion follows from Lemma 2 .

Lemma 3. The operator $A_{\epsilon}$ is monotone, meaning

$$
\left(A_{\epsilon}(u)-A_{\epsilon}(v), \nabla u-\nabla v\right) \geq 0 .
$$


When $u, v \in W_{0}^{1, M_{\epsilon}}(\Omega)$, equality only holds if $u=v$.

Proof. The increasing character of $F_{\epsilon}$ means

$$
(y-x) F_{\epsilon}(x) \leq(y-x) F_{\epsilon}(y),
$$

and equality only holds if $x=y$. Therefore,

$$
\left(\mu_{\epsilon}(x)+\mu_{\epsilon}(y)\right) x y \leq \mu_{\epsilon}(x) x^{2}+\mu_{\epsilon}(y) y^{2}
$$

with the same caveat. Hence

$$
\begin{array}{r}
\int_{\Omega}\left(\mu_{\epsilon}(|\nabla u|) \nabla u-\mu_{\epsilon}(|\nabla v|) \nabla v\right) \cdot(\nabla u-\nabla v) d x \\
\geq \int_{\Omega} \mu_{\epsilon}(|\nabla u|)|\nabla u|^{2}+\mu_{\epsilon}(|\nabla v|)|\nabla v|^{2} \\
-\left(\mu_{\epsilon}(|\nabla u|)+\mu_{\epsilon}(|\nabla v|)\right)|\nabla u||\nabla v| d x \geq 0 .
\end{array}
$$

Equality demands $|\nabla u|=|\nabla v|, \nabla u \cdot \nabla v=|\nabla u||\nabla v|$; thus, $\nabla u=\nabla v$ almost everywhere in $\Omega$. Since both $u$ and $v$ vanish at $\partial \Omega$, they are identical.

Lemma 4. Let $u \in W_{0}^{1, M}(\Omega)$. Then

$$
\frac{1}{\|\nabla u\|_{L^{M_{\epsilon}}}} \int_{\Omega} \mu_{\epsilon}(|\nabla u|)|\nabla u|^{2} d x=\frac{1}{\|\nabla u\|_{L^{M_{\epsilon}}}}\left(A_{\epsilon}(u), \nabla u\right)
$$

tends to $\infty$ when $\|\nabla u\|_{L^{M_{\epsilon}}} \rightarrow \infty$.

Proof. We will use the so-called Luxemburg norm in $L^{M_{\epsilon}}$, which is equivalent to the standard one; we will denote it by $\|u\|_{M_{\epsilon}}$. Since $N_{\epsilon}$ satisfies the $\Delta_{2}$ condition, we have

$$
\int_{\Omega} M_{\epsilon}\left(\frac{|\nabla u|}{\|\nabla u\|_{M_{\epsilon}}}\right) d x=1
$$

(see Ref. 13, p. 78). Therefore,

$$
1=\int_{\Omega} \mu_{\epsilon}\left(\frac{|\nabla u|}{\|\nabla u\|_{M_{\epsilon}}}\right) \frac{|\nabla u|^{2}}{\|\nabla u\|_{M_{\epsilon}}^{2}} d x
$$

Since $\mu_{\epsilon}$ is increasing, when $\|\nabla u\|_{M_{\epsilon}}$ grows we obtain

$$
\|\nabla u\|_{M_{\epsilon}} \leq \frac{1}{\|\nabla u\|_{M_{\epsilon}}} \int_{\Omega} \mu_{\epsilon}(|\nabla u|)|\nabla u|^{2} d x,
$$

which proves the result.

Recall that the function $u \in W_{0}^{1, M_{\epsilon}}(\Omega)$ is called a weak solution of the problem

$$
\begin{array}{r}
\nabla \cdot\left(\mu_{\epsilon}(|\nabla u|) \nabla u\right)=\rho, \\
\left.u\right|_{\partial \Omega}=0,
\end{array}
$$

if for every $v \in W_{0}^{1, M_{\epsilon}}(\Omega)$, the relation

$$
\int_{\Omega} \mu_{\epsilon}(|\nabla u|) \nabla u \cdot \nabla v d x=-\int_{\Omega} \rho v d x
$$

holds.

We state the main theorem: the standard MOND case corresponds to $\epsilon=0$.

Theorem 1: For every $\epsilon \in[0,1]$, the mapping

$$
\begin{aligned}
T_{\epsilon}: W_{0}^{1, M_{\epsilon}}(\Omega) & \rightarrow\left(W_{0}^{1, M_{\epsilon}}(\Omega)\right)^{\prime} \\
T_{\epsilon}(u)(v) & =\left(A_{\epsilon}(u), \nabla v\right)
\end{aligned}
$$


is an isomorphism. Therefore, for every $\rho$ in the dual space $\left(W_{0}^{1, M_{\epsilon}}(\Omega)\right)^{\prime}$ there exists a unique weak solution of (33).

Proof. Lemmas 1-3 show that $T_{\epsilon}$ satisfies the conditions in p. 171-173 of Ref. 16. The result follows.

For the non-homogeneous Dirichlet problem

$$
\begin{array}{r}
\nabla \cdot\left(\mu_{\epsilon}(|\nabla u|) \nabla u\right)=\rho, \\
\left.u\right|_{\partial \Omega}=g,
\end{array}
$$

we must assume the existence of an extension of $g$ to a certain $w \in W_{0}^{1, M_{\epsilon}}(\Omega)$. Defining $v=u-w$, we must solve

$$
\begin{array}{r}
\nabla \cdot\left(\mu_{\epsilon}(|\nabla(v+w)|) \nabla(v+w)\right)=\rho, \\
\left.v\right|_{\partial \Omega}=0 .
\end{array}
$$

It is easy to check that the operator

$$
B_{\epsilon}(v)=A_{\epsilon}(v+w)
$$

satisfies the same properties as $A_{\epsilon}$, so there exists again a unique solution.

\section{REGULARITY OF THE MOND POTENTIALS}

There exist two problems with these apparently general theorems. The first one is that while

$$
L^{\infty}(\Omega) \subset L^{M_{\epsilon}}(\Omega) \subset L^{1}(\Omega)
$$

and therefore

$$
L^{\infty}(\Omega) \subset\left(W_{0}^{1, M_{\epsilon}}(\Omega)\right)^{\prime} \subset M(\Omega),
$$

it is difficult to precise exactly the admissible densities. Certainly Dirac measures are not there, so we cannot use point masses. This, however, is more a problem of convenience than a physical one; we can always assume that densities are bounded measurable functions. It would be unreasonable to demand more; solid planets yield a noncontinuous density in the Solar System.

The second problem is that the pertinence of the MOND potential to $W^{1, M_{\epsilon}}(\Omega)$ does not say a lot about the regularity properties of it. In the classical theory of linear and quasilinear elliptic equations, the more regular is the independent term the more regular is the solution; thus, when $\rho \in L^{\infty}(\Omega)$, the solution $u$ belongs to a Hölder class $\mathcal{C}^{0, \alpha}(\Omega) .{ }^{17,18}$ Even this is not entirely acceptable, since we would like the gradient of $u$, which is the real physical force, to be at least bounded. This is partially solved by the fact that these results are local; if $\rho$ is smooth enough in a domain, so is $u$ in any subdomain whose closure is contained there.

For the MOND case, however, the lack of ellipticity is a major obstacle. However, we must recall that MOND dynamics are relevant when the size of $\nabla u$ is small (i.e., when the equation is less elliptic); even for moderately large gravitational fields, it coincides very precisely with Newtonian predictions. If we make the hypothesis that within our region of interest $|\nabla u| \leq R$, for any given constant $\delta$ we may take $\epsilon$ small enough $\left(\epsilon R^{2} \leq \delta\right)$ for

$$
\left|\mu_{\epsilon}(|\nabla u|)-\mu(|\nabla u|)\right| \leq \delta,
$$

and presume that the MOND potential and the $\epsilon$-MOND one will differ very little. With this admittedly pragmatical hypothesis in place, we may obtain nice regularity results.

Theorem 2: Assume that $\rho$ is measurable and bounded in a region $\Omega$. For any $\epsilon \in(0,1]$ and any relatively compact domain $\Omega^{\prime}$ such that $\overline{\Omega^{\prime}} \subset \Omega$, there exists $\alpha>0$ such that the solution to (31) lies in the space $\mathcal{C}^{1, \alpha}\left(\Omega^{\prime}\right)$. 
Proof. The vector

$$
\mathbf{a}(\mathbf{x}, z, \mathbf{p})=\mu_{\epsilon}(|\mathbf{p}|) \mathbf{p}
$$

satisfies

$$
\begin{gathered}
\mathbf{a}(\mathbf{x}, z, \mathbf{0})=\mathbf{0} \\
\sum_{i, j} \frac{\partial a_{j}}{\partial p_{i}} \xi_{i} \xi_{j}=\left(\epsilon+(1-\epsilon) \mu^{\prime}(|\mathbf{p}|)\right) \frac{(\mathbf{p} \cdot \boldsymbol{\xi})^{2}}{|\mathbf{p}|} \\
+\epsilon|\mathbf{p}| \xi^{2}+(1-\epsilon) \mu(|\mathbf{p}|) \xi^{2} \geq \epsilon|\mathbf{p}| \xi^{2} \\
\sum_{i, j}\left|\frac{\partial a_{j}}{\partial p_{i}}\right|=\sum_{i, j}\left(\epsilon \frac{p_{i} p_{j}}{|\mathbf{p}|}+(1-\epsilon) \mu^{\prime}(|\mathbf{p}|) \frac{p_{i} p_{j}}{|\mathbf{p}|}\right) \\
+3 \epsilon|\mathbf{p}|+3(1-\epsilon) \mu(|\mathbf{p}|) \leq K|\mathbf{p}|,
\end{gathered}
$$

for some constant $K$. Then we may apply Theorem 1 of Ref. 19 to obtain the result. (The remaining conditions are trivially satisfied. Notice that there is a misprint in Eq. (1.4) of this paper.)

Thus, the solutions to the $\epsilon$-MOND equation are more regular than those of the Newtonian Poisson equation. In particular $\nabla u \in \mathcal{C}^{0, \alpha}$, so the gradient is continuous. That this cannot be improved is shown by the well known spherical solution

$$
u(\mathbf{x})=\frac{3}{2}|\mathbf{x}|^{3 / 2},
$$

which satisfies

$$
\nabla \cdot(|\nabla u| \nabla u)=3 .
$$

\section{QUASILINEAR MOND}

This new version of MOND ${ }^{11}$ satisfies the following system. First take the Newtonian potential

$$
\Delta u^{N}=\rho,
$$

and then consider the new Poisson equation

$$
\Delta u=\nabla \cdot\left(v\left(\frac{\left|\nabla u^{N}\right|}{a_{0}}\right) \nabla u^{N}\right) .
$$

$v$ is related to the function $\mu$ by $v(y)=1 / \mu(x)$, where $x \mu(x)=y$. For the function $\mu$ given in (3), this yields

$$
v(y)=1+\frac{2}{y+\sqrt{y^{2}+4 y}},
$$

so that $v(y) \sim 1 / \sqrt{y}$ when $y \rightarrow 0$ and $\nu(y) \rightarrow 1$ when $y \rightarrow \infty$. It is not clear if we must take different boundary conditions for $u^{N}$ and $u$; presumably they must be taken as zero when considering a large domain, with no matter near the boundary. Anyway, our bounds will hold as long as the boundary conditions are smooth enough.

Theorem 3: Assume that $\rho \in L^{p}(\Omega), p \in(1, \infty)$. For $p<3, u \in W^{1, q}(\Omega)$, with $q=6 p /(3-p)$. Therefore, $u \in \mathcal{C}^{0, \alpha}(\Omega)$ for $\alpha=3(p-1) /(2 p)$. For $p \geq 3, u \in W^{1, r}(\Omega)$ for any $r<\infty$, so that $u \in \mathcal{C}^{0, \alpha}(\Omega)$ for any $\alpha<1$. 
Proof. For $\rho \in L^{p}(\Omega), p \in(1, \infty)$, it is well known that the Newtonian potential $u^{N} \in W^{2, p}(\Omega){ }^{20}$ Using Sobolev's injection lemmas,

$$
\begin{aligned}
& \left|\nabla u^{N}\right| \in L^{p^{*}}(\Omega), \quad p^{*}=\frac{3 p}{3-p}, \quad \text { if } p<3 \\
& \left|\nabla u^{N}\right| \in L^{r}(\Omega), \quad \text { for any } r<\infty \quad \text { if } p \geq 3 .
\end{aligned}
$$

Since $v(y) \leq k / \sqrt{y}$ for some constant $k$,

$$
v\left(\left|\nabla u^{N}\right|\right)\left|\nabla u^{N}\right| \leq k\left|\nabla u^{N}\right|^{-1 / 2}\left|\nabla u^{N}\right| \in L^{2 p^{*}}(\Omega) \text { if } p<3,
$$

or any $L^{r}, r<\infty$, if $p \geq 3$. Therefore

$$
\nabla \cdot\left(v\left(\left|\nabla u^{N}\right|\right) \nabla u^{N}\right) \in\left(W_{0}^{1,2 p^{*}}(\Omega)\right)^{\prime},
$$

for $p<3$. Notice that $2 p^{*}=(6 p) /(3-p)$. For $p \geq 3$, any $r<\infty$ is valid. By the classical theorems on the linear Poisson equation, for smooth enough boundary conditions, $u \in W^{1,2 p^{*}}(\Omega)$ (respectively $u \in W^{1, r}(\Omega)$, any $\left.r<\infty\right)$. The remaining points follow from Sobolev's lemmas on the imbedding of Sobolev in Hölder spaces.

We see that quasilinear MOND yields less regularity than Newtonian dynamics, but still enough to guarantee a physically admissible behaviour.

\section{CONCLUSIONS}

Modified Newtonian dynamics is an unconventional proposal to explain the observed behaviour of galaxies' rotation without recourse to the presence of dark matter. It is still an active object of research in the astrophysical community, but the mathematics of the basic nonlinear Poisson equation has been rather neglected. This equation is problematic as it lies halfway between strictly elliptic quasilinear equations and the p-Laplace equation, being neither one nor the other, so that none of the usual existence theorems apply directly. To prove this existence, we need to define an appropriate Orlicz-Sobolev space for an Orlicz function directly related to the Milgrom function which scales the effect of the gravitational force. The resulting existence and uniqueness theorem is somewhat unsatisfactory as it provides for a wide class of admissible densities and does not yield much regularity of the solutions. However, we also show that in the regions where MOND differs substantially from Newtonian mechanics, i.e. where the gravitational force is low, we may approximate the MOND equation by a Poisson-like equation that yields for bounded densities a gravitational field whose gradient is Hölder continuous, which represents a higher degree of regularity than the Newtonian one. A later proposal, quasilinear MOND, is also studied.

\section{ACKNOWLEDGMENTS}

Partially supported by the Ministry of Economy and Competitiveness of Spain (Contract No. MTM200912561).

\footnotetext{
${ }^{1}$ M. Milgrom, "A modification of the Newtonian dynamics as a possible alternative to the hidden mass hypothesis," Astrophys. J. 270, 365-370 (1983).

${ }^{2}$ M. Milgrom, "A modification of the Newtonian dynamics: implications for galaxies," Astrophys. J. 270, 371-383 (1983).

${ }^{3}$ R. H. Sanders and S. S. McGaugh, "Modified Newtonian dynamics as an alternative to dark matter," Annu. Rev. Astron. Astrophys. 40, 263-317 (2002).

${ }^{4}$ J. D. Bekenstein, "Relativistic gravitation theory for the modified Newtonian dynamics paradigm," Phys. Rev. D 70, 083509 (2004).

${ }^{5}$ R. H. Sanders, "A tensor-vector-scalar framework for modified dynamics and cosmic dark matter,” Mon. Not. R. Astron. Soc. 363, 459-468 (2005).

${ }^{6}$ D. Giannios, "Spherically symmetric, static spacetimes in a tensor-vector-scalar theory," Phys. Rev. D 71, 103511 (2005).

${ }^{7}$ G. W. Angus, B. Famaey, and H. S. Zhao, "Can MOND take a bullet? Analytical comparisons of three versions of MOND beyond spherical symmetry," Mon. Not. R. Astron. Soc. 371, 138-146 (2006).

${ }^{8}$ C. R. Contaldi, T. Wiseman, and B. Withers, "TeVeS gets caught on caustics," Phys. Rev. D 78, 044034 (2008).
} 
${ }^{9}$ M. Milgrom, "Solutions for the modified Newtonian dynamics equation," Astrophys. J. 302, 617-625 (1986).

${ }^{10}$ R. Brada and M. Milgrom, "Exact solutions and approximations of MOND fields and disc galaxies," Mon. Not. R. Astron. Soc. 276, 453-459 (1995).

${ }^{11}$ M. Milgrom, "Quasi-linear formulation of MOND,” Mon. Not. R. Astron. Soc. 403, 886-895 (2010).

${ }^{12}$ J. D. Bekenstein and J. Magueijo, "Modified Newtonian dynamics within the solar system," Phys. Rev. D 73, 103513 (2006).

${ }^{13}$ M. A. Krasnosel'skii and Ya. B. Rutickii, Convex Functions and Orlicz Spaces (Noordhoff, Groningen, Netherlands, 1961).

${ }^{14}$ A. Kufner, O. John, and S. Fucik, Function Spaces (Noordhoff, Leyden, Netherlands, 1977).

${ }^{15}$ M. M. Rao and Z. D. Ren, Applications of Orlicz Spaces (Marcel Dekker, New York, 2002).

${ }^{16}$ J. L. Lions, Quelques méthodes de résolution des problémes aux limites non linéaires (Dunod, Paris, 1969).

${ }^{17}$ O. A. Ladyzhenskaya and N. N. Ural'tseva, Linear and Quasilinear Elliptic Equations (Academic Press, New York, 1968) [Russian Edition, Nauka, Moscow (1964)].

${ }^{18}$ J. Serrin, "Local behavior of solutions of quasilinear equations," Acta Math. 111, 247-302 (1964).

${ }^{19}$ P. Tolksdorf, "Regularity for a more general class of quasilinear elliptic equations," J. Differ. Equations 51, 126-150 (1984).

${ }^{20}$ D. Gilbarg and N. S. Trudinger, Elliptic Partial Differential Equations of Second Order (Springer, New York, 1998). 